\title{
SU(2) COHERENT STATE PATH INTEGRAL INVESTIGATION OF THE HOLSTEIN DIMER
}

\author{
T. HakioğLU ${ }^{a}$, V.A. IVAnov ${ }^{b}$ and M.Ye. Zhuravlev ${ }^{b}$ \\ ${ }^{a}$ Bilkent University, Department of Physics, 06533 Ankara, Turkey \\ ${ }^{b}$ N.S. Kurnakov Institute of General and Inorganic Chemistry of the RAS \\ 117907 Moscow, Russia
}

The SU(2) coherent state path integral is used to investigate the partition function of the Holstein dimer. This approach naturally takes into account the dynamical symmetry of the model. The ground-state energy and the number of the phonons are calculated as functions of the parameters of the Hamiltonian. The renormalizations of the phonon frequency and electron hopping for bonding and antibonding states are considered. The destruction of quasiclassical mean field solution is discussed.

PACS numbers: 03.65.Db, 71.36.+c, 64.60.Ak

\section{Introduction}

Despite their simplicity, the dimer models are being intensively investigated because they represent simple interacting electron-phonon systems which can provide information about the polaron dynamics for more realistic but equally unsolvable systems. There are various analytical (semi-analytical) approaches to the electron-phonon systems with a few number of electrons interacting with local vibrations $[1,2]$. In the present work we examine a different method in which the $\mathrm{SU}(2)$ coherent state path integral is applied to calculate analytically the partition function of the Holstein dimer with a single electron. This method naturally incorporates the dynamical symmetry of the underlying Hamiltonian.

\section{2. $\mathrm{SU}(2)$ coherent state path integral representation of the partition function of the Holstein dimer}

We start with the one-mode Hamiltonian

$$
\begin{aligned}
& H_{\mathrm{dim}}=-t\left(c_{1}^{+} c_{2}+c_{2}^{+} c_{1}\right)+\omega\left(a_{1}^{+} a_{1}+a_{2}^{+} a_{2}\right) \\
& \quad+g\left(a_{1}^{+}+a_{1}\right) n_{1}+g\left(a_{2}^{+}+a_{2}\right) n_{2}+\epsilon\left(n_{1}-n_{2}\right)
\end{aligned}
$$

with the one-electron constraint $n_{1}+n_{2}=1$ where in the case of no spin-dependent interaction we drop the spin indices from the fermion operators. We can separate in Eq. (1) one phonon degree of freedom by rotating the initial phonon coordinates as

$$
a_{1}=(w+v) / \sqrt{2}, \quad a_{2}=(-w+v) / \sqrt{2} .
$$


The initial Hamiltonian is then written in a separable form as

$$
H_{\text {dim }}=H_{v}+H_{w} \text {, }
$$

where

$$
\begin{aligned}
& H_{v}=\omega v^{+} v+\frac{g}{\sqrt{2}}\left(v^{+}+v\right) \\
& H_{w}=-t\left(c_{1}^{+} c_{2}+c_{2}^{+} c_{1}\right)+\omega w^{+} w+\left[\frac{g}{\sqrt{2}}\left(w^{+}+w\right)+\epsilon\right]\left(n_{1}-n_{2}\right) .
\end{aligned}
$$

The partition function of the Hamiltonian (1) is the product of the partition function $Z_{\nu}$ of the displaced harmonic oscillator (2) and the partition function $Z_{w}$ of the Hamiltonian (3). The partition function $Z_{w}$ cannot be calculated exactly. Due to the one-electron constraint the Hamiltonian (3) can be naturally rewritten by using the electron pseudospin operators in the representation $\hat{J}_{+}=c_{1}^{+} c_{2}$, $\hat{J}_{-}=c_{2}^{+} c_{1}, \hat{J}_{0}=\frac{1}{2}\left(c_{1}^{+} c_{1}-c_{2}^{+} c_{2}\right)$. Applying the rotation in the spin space $\hat{J}_{y} \rightarrow \hat{J}_{0}$, $\hat{J}_{z} \rightarrow-\hat{J}_{y}$ we then obtain the following form of the spin Hamiltonian:

$$
\begin{aligned}
H_{w} & =\omega w^{+} w-\left\{t-\mathrm{i}\left[\epsilon+\frac{g}{\sqrt{2}}\left(w^{+}+w\right)\right]\right\} \hat{J}_{+} \\
& -\left\{t+\mathrm{i}\left[\epsilon+\frac{g}{\sqrt{2}}\left(w^{+}+w\right)\right]\right\} \hat{J}_{-} .
\end{aligned}
$$

Following [3] we apply the SU(2) coherent state path integral to represent the partition function of the Hamiltonian (4) as

$$
\begin{aligned}
Z_{w}= & \operatorname{Tr} \mathrm{e}^{-\beta H_{w}}=\int \mathrm{D} w \mathrm{D} \bar{w} \mathrm{D} \mu_{\mathrm{SU}(2)} \exp \left\{\int_{0}^{\beta}[-\bar{w} \dot{w}-\omega \bar{w} w\right. \\
& +\frac{1}{2} \frac{\dot{\bar{\alpha}}(\tau) \alpha(\tau)-\bar{\alpha}(\tau) \dot{\alpha}(\tau)}{1+|\alpha|^{2}}+\frac{1}{2}\left[t+\mathrm{i}\left(\epsilon+\frac{g}{\sqrt{2}}(\bar{w}(\tau)+w(\tau))\right)\right] \frac{\alpha(\tau)}{1+|\alpha|^{2}} \\
& \left.\left.+\frac{1}{2}\left[t-\mathrm{i}\left(\epsilon+\frac{g}{\sqrt{2}}(\bar{w}(\tau)+w(\tau))\right)\right] \frac{\bar{\alpha}(\tau)}{1+|\alpha|^{2}}\right] \mathrm{~d} \tau\right\}
\end{aligned}
$$

The path integral over the $\mathrm{SU}(2)$ variables can be calculated by the method developed in $[3,4]$. Following $[3,4]$ the $\mathrm{SU}(2)$ path integration yields

where

$$
\begin{aligned}
Z_{w} & =Z_{w-}+Z_{w+}=\int \mathrm{D} w \mathrm{D} \bar{w} \exp \left(S_{-}\right)+\int \mathrm{D} w \mathrm{D} \bar{w} \exp \left(S_{+}\right) \\
& =\int \mathrm{D} w \mathrm{D} \bar{w} \exp \int_{0}^{\beta}(-\bar{w} \dot{w}+\omega \bar{w} w+\Omega / 2) \mathrm{d} \tau \\
& +\int \mathrm{D} w \mathrm{D} \bar{w} \exp \int_{0}^{\beta}(-\bar{w} \dot{w}+\omega \bar{w} w-\Omega / 2) \mathrm{d} \tau,
\end{aligned}
$$

$$
\begin{aligned}
\Omega(\tau) & =-\left\{t+\mathrm{i}\left[\epsilon+\frac{g}{\sqrt{2}}(\bar{w}(\tau)+w(\tau))\right]\right\} z(\tau) \\
& -\left\{t-\mathrm{i}\left[\epsilon+\frac{g}{\sqrt{2}}(\bar{w}(\tau)+w(\tau))\right]\right\} \bar{z}(\tau) .
\end{aligned}
$$

The auxiliary functions $z(\tau), \bar{z}(\tau)$ should be defined through the system of differ- 
ential Riccati equations [4], which cannot be solved exactly as a functional of the arbitrary functions $\vec{w}(\tau), w(\tau)$. We will, in the following, investigate the partition function (6) in the stationary phase approximation.

\section{Stationary phase approximation}

In accordance with the general scheme of stationary phase approximation, the partition function $Z_{w \pm}$ is represented as

$$
\int \mathrm{D} \bar{w} \mathrm{D} w \mathrm{e}^{S} \sim \mathrm{e}^{S_{0}} \int \mathrm{D} \bar{w} \mathrm{D} w \mathrm{e}^{\delta^{2} S}=\mathrm{e}^{S_{0}}(\operatorname{Det} L)^{-1},
$$

where we denote by $S_{0}$ the zeroth order term (the stationary solution) for the action $S$. Here $L$ is the kernel of the non-linear integral operator defined by the second variation of the action (6).

As a result we obtain the following expression for the partition function of the initial system (1):

$$
\begin{aligned}
& Z=Z_{+}+Z_{-} \\
& Z_{ \pm}=Z_{v} \cdot Z_{w \pm}=\frac{\mathrm{e}^{-\beta E_{ \pm}}}{\left(1-\mathrm{e}^{-\beta \omega}\right)\left(1-\mathrm{e}^{-\beta \omega_{1-}}\right)} \frac{1-\mathrm{e}^{-2 \beta E_{0}}}{1-\mathrm{e}^{-\beta \omega_{2-}}} .
\end{aligned}
$$

Here

$$
\begin{aligned}
& E_{ \pm}=\frac{\omega W_{0-}^{2}}{4}-(1 \mp 1) E_{0}+\frac{\omega_{1}+\omega_{2}-\omega}{2}-\frac{g^{2}}{2 \omega}, \\
& E_{0}=\sqrt{t^{2}+\left(\epsilon+\frac{g}{\sqrt{2}} W_{0-}\right)^{2}}, \\
& \omega_{1-, 2-}^{2}=\frac{\left(4 E_{0}^{2}+\omega^{2}\right) \pm \sqrt{\left(4 E_{0}^{2}-\omega^{2}\right)^{2}+\frac{16 g^{2} t^{2} \omega}{E_{0}}}}{2}, \\
& \omega_{1+, 2+}^{2}=\frac{\left(4 E_{0}^{2}+\omega^{2}\right) \pm \sqrt{\left(4 E_{0}^{2}-\omega^{2}\right)^{2}-\frac{16 g^{2} t^{2} \omega}{E_{0}}}}{2} .
\end{aligned}
$$

The condition for $W_{0 \pm}$ is given by

$$
W_{0 \pm}=\mp \frac{\sqrt{2} g\left(\epsilon+\frac{g}{\sqrt{2}} W_{0 \pm}\right)}{\omega E_{0} \operatorname{sgn}\left(\epsilon+\frac{g}{\sqrt{2}} W_{0 \pm}\right)} .
$$

\section{Discussion and conclusion}

We investigate the partition function of the dimer (1) in the quasiclassical approximation. The region of validity of the stationary phase approximation cannot be defined exactly. The calculated ground state energy $E_{-}(8)$ contains the contributions from the stationary trajectories and from determinant (quantum fluctuations). The general opinion is that the approximation is adequate if the contribution from the stationary trajectories is dominant. 
The ratio of these contributions is represented in Fig. 1. Another physical quantity which can be calculated with the help of the obtained partition function is the thermal phonon occupation factor $n_{\mathrm{ph}}=-\frac{1}{Z \beta} \frac{\mathrm{d} Z}{\mathrm{~d} \omega}$ which is presented in Fig. 2 for such low temperature $(\beta t=10000)$ that it is sufficient to consider only the contribution from the ground state energy. In the narrow interval of the electron-phonon coupling constant $g$, where the mentioned ratio shows the highest values, our approach is inapplicable and the number of the phonons becomes negative. In this region the quantum fluctuations destroy the mean field. We suppose that the smooth transition to self-trapping of electron takes place in this area.
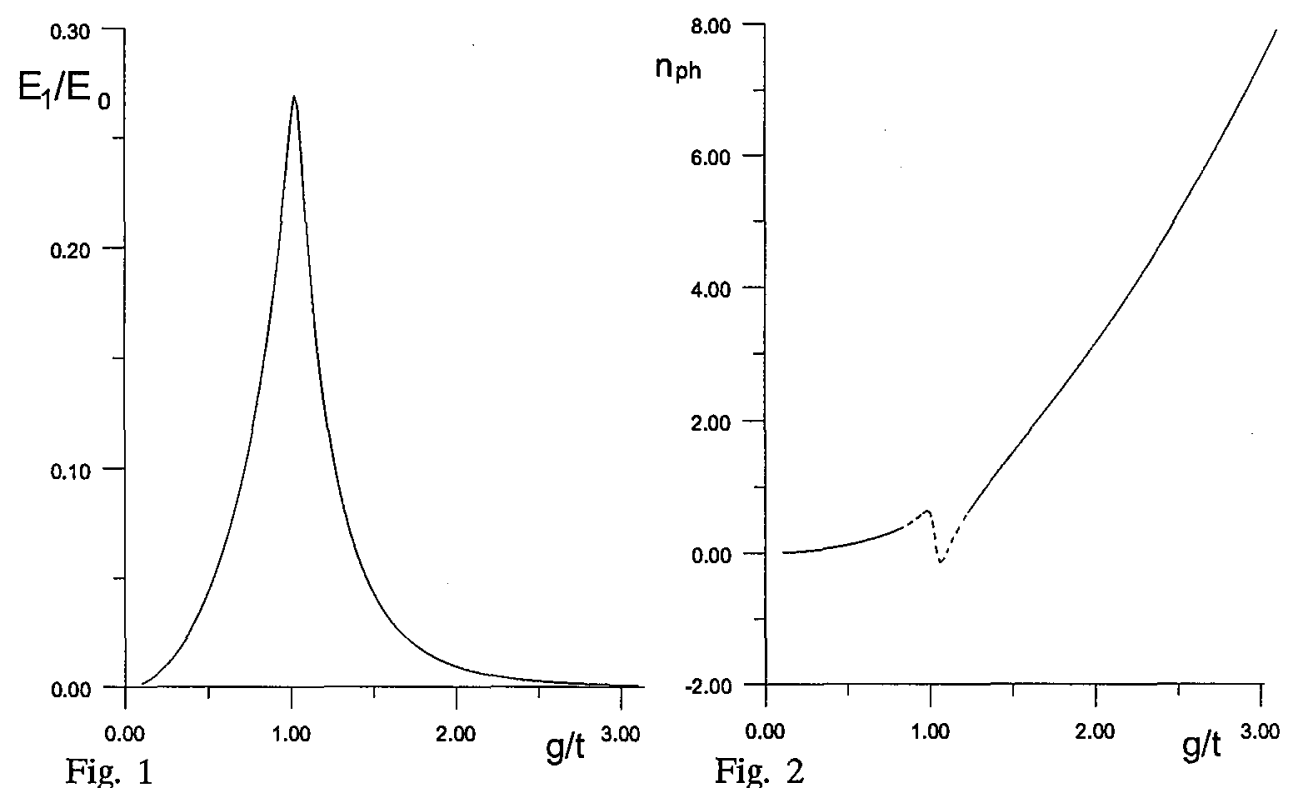

Fig. 1

Fig. 2

Fig. 1. The ratio of the contributions into the ground state energy from determinant $\left(E_{1}\right)$ and stationary trajectories $\left(E_{0}\right)$ for $\omega / t=0.6$.

Fig. 2. Number of phonons for $\omega / t=0.6$. Non-physical region where mean field is destroyed by quantum fluctuations is marked by dashed line.

Another quantity which is often calculated is the renormalized phonon frequency. Our analysis shows (see (7)) that a realistic picture is more complicated than a simple renormalization of the initial phonon frequency (compare with [5]).

\section{References}

[1] A.S. Alexandrov, V.V. Kabanov, D.K. Ray, Phys. Rev. B 49, 9915 (1994).

[2] J. Ranninger, U. Thibblin, Phys. Rev. B 45, 7730 (1992).

[3] E.A. Kochetov, J. Math. Phys. 36, 4667 (1995).

[4] E.A. Kochetov, Phys. Rev. B 52, 4402 (1995).

[5] R. Pucci, V.S. Yarunin, M.Ye. Zhuravlev, J. Phys. A 31, 3185 (1998). 